\title{
Les bovins de la fosse médiévale deTiya (Éthiopie) : dépôt rituel ou banquet funéraire?
}

\author{
Joséphine Lesur
}

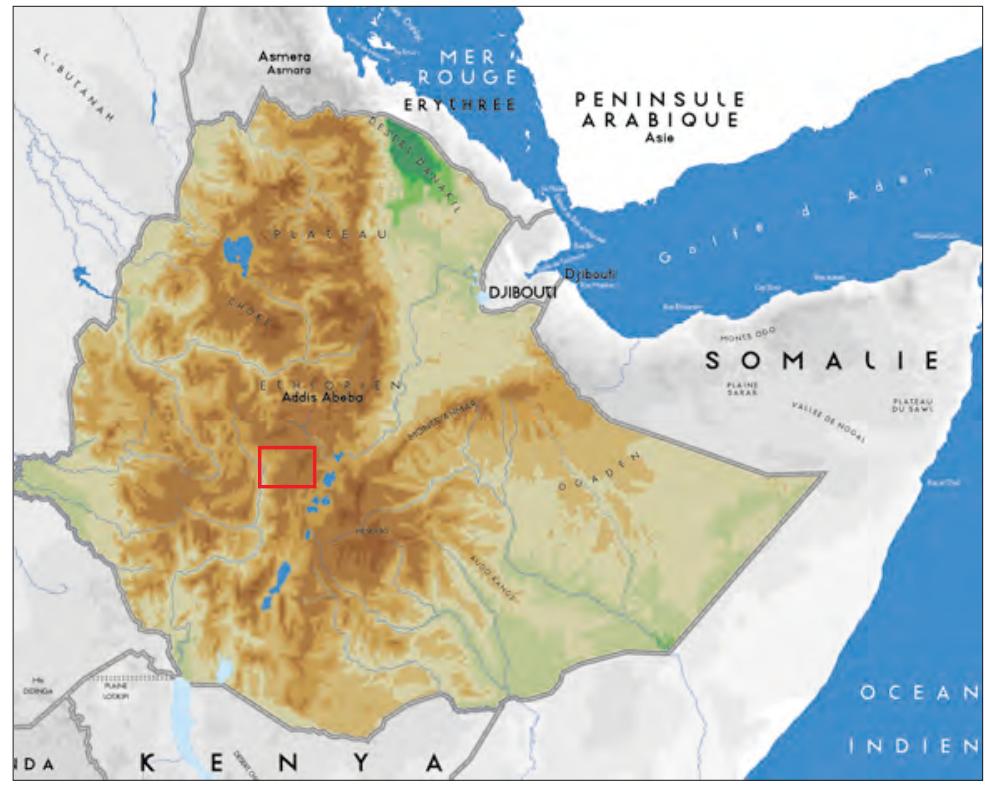

\section{RÉSUMÉ}

L'analyse archéozoologique des restes fauniques de la Fosse médiévale de Tiya (Éthiopie) a révélé la présence exclusive de bovins. Au moins quatre individus adultes ont été retrouvés comprenant probablement des mâles, des femelles et des castrés. Les résultats de l'analyse attestent d'un abattage et d'une consommation des animaux sur place. L'association de ces résultats avec les données anthropologiques provenant de l'étude du cimetière à stèles contigu à la fosse, suggèrent un lien possible entre l'utilisation des bovins et les inhumations.

Mots clés : archéozoologie, Corne de l'Afrique, bovins, pratiques funéraires.

\section{Abstract}

The archaeozoological analysis of the faunal remains of the medieval pit of Tiya (Ethiopia) revealed the exclusive presence of bovines. At least four adult animals were found, including males, females and castrated. Results of the analysis testify the kill and the consumption of the animals in situ. These results associated with the anthropological data coming from the stele cemetery adjoining the pit, suggest a possible link between the use of the bovines and the funeral.

Keywords: archaeozoology, Horn of Africa, bovines, funerary practices. 
Situé à une centaine de kilomètres au sud d'Addis Abeba (Ethiopie), dans la région du Soddo (figure 1), le site à stèles de Tiya a été classé au patrimoine mondial par l'UNESCO en 1980. A partir de 1982 et jusqu'en 1992, R. Joussaume accompagné d'une équipe d'archéologues a fouillé le site qui se compose d'une quarantaine de stèles répartie en trois ensembles (Joussaume, 1995). Ces stèles, dont la plus haute atteint $5 \mathrm{~m}$, marquent des tombes en fosses circulaires plus ou moins profondes qui peuvent contenir, posés sur le fond, jusqu'à trois individus. La majorité des stèles sont décorées d'épées en champlevé, au nombre variable, situées au dessus d'un ensemble de symboles toujours identiques et d'une perforation basale qui pourrait être en rapport avec le mort dans sa tombe. En plus de ces tombes à stèles, d'autres sépultures ont été dégagées en arrière des stèles, formant des lignes sur trois ou quatre rangs, suggérant un possible lien familial (Joussaume, 1995). D'après les datations, ce cimetière aurait fonctionné entre le $\mathrm{XI}^{\mathrm{e}}$ et le $\mathrm{XIII}^{\mathrm{e}}$ siècle de notre ère.

Dans la partie nord du site, une fosse, découverte en 1982, a livré un matériel archéologique abondant composé notamment d'ossements de bovins. Il semble que cette fosse soit contemporaine du cimetière, suggérant une possible relation entre eux.

Grâce à l'analyse détaillée des vestiges fauniques, nous tenterons tout d'abord d'apporter de nouveaux éléments sur les bovins protohistoriques éthiopiens et, d'une manière plus générale, sur l'origine et le développement de l'élevage dans la Corne de l'Afrique.
Dans un second temps, nous essayerons de comprendre quelle pouvait être la nature du lien entre le cimetière et l'utilisation de ces animaux : s'agit-il de restes d'un banquet funéraire donné à l'occasion de l'enterrement d'un des occupants du cimetière ? Ou sommes nous en présence d'un dépôt rituel lié à la mort et aux croyances religieuses de cette population ? Enfin, si le cimetière et la fosse ne sont pas associés, alors peuton rattacher cette dernière à d'autres structures archéologiques, peut-être d'habitation?

\section{Présentation de la FOSSE}

Au nord du principal ensemble de tombes, R. Joussaume et son équipe ont dégagé en 1982 une cuvette artificielle contenant de nombreuses pierres, fragments osseux et tessons de poterie (figure 2). En 1990, la fouille a été élargie afin d'obtenir une vision d'ensemble de cette structure (Joussaume, 1995).

Cette fosse "offre un contour sinueux et irrégulier, grossièrement quadrangulaire, d'une dimension moyenne de 5,80 $m x$ $4,20 \mathrm{~m}$. Elle présente en coupe une légère déclivité sur le pourtour, accentuée à une distance variable, jusqu'à $1 \mathrm{~m}$ de son bord, avec un fond presque plat et une profondeur d'environ 1,10 $\mathrm{m}$ par rapport au sol actuel. " (Lagasquie, 1995: 276) Elle est creusée dans la couche profonde du sous-sol, celle-là même qui

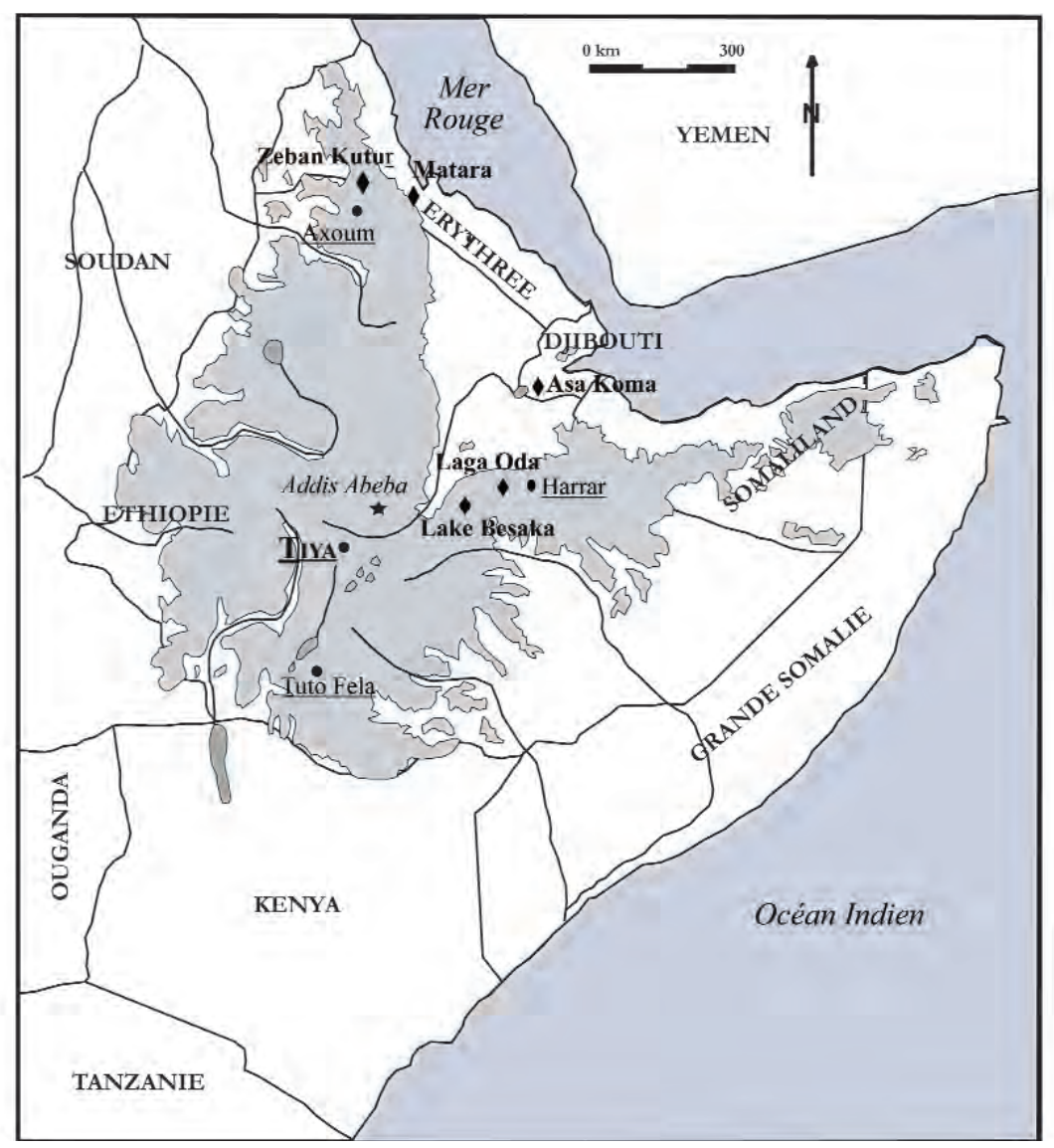

Figure 1 - La corne de l'Afrique : Éthiopie, Somalie, Somaliland, Djibouti et Érythrée. Localisation de TIYA, de quelques sites mégalithiques et archéologiques. 


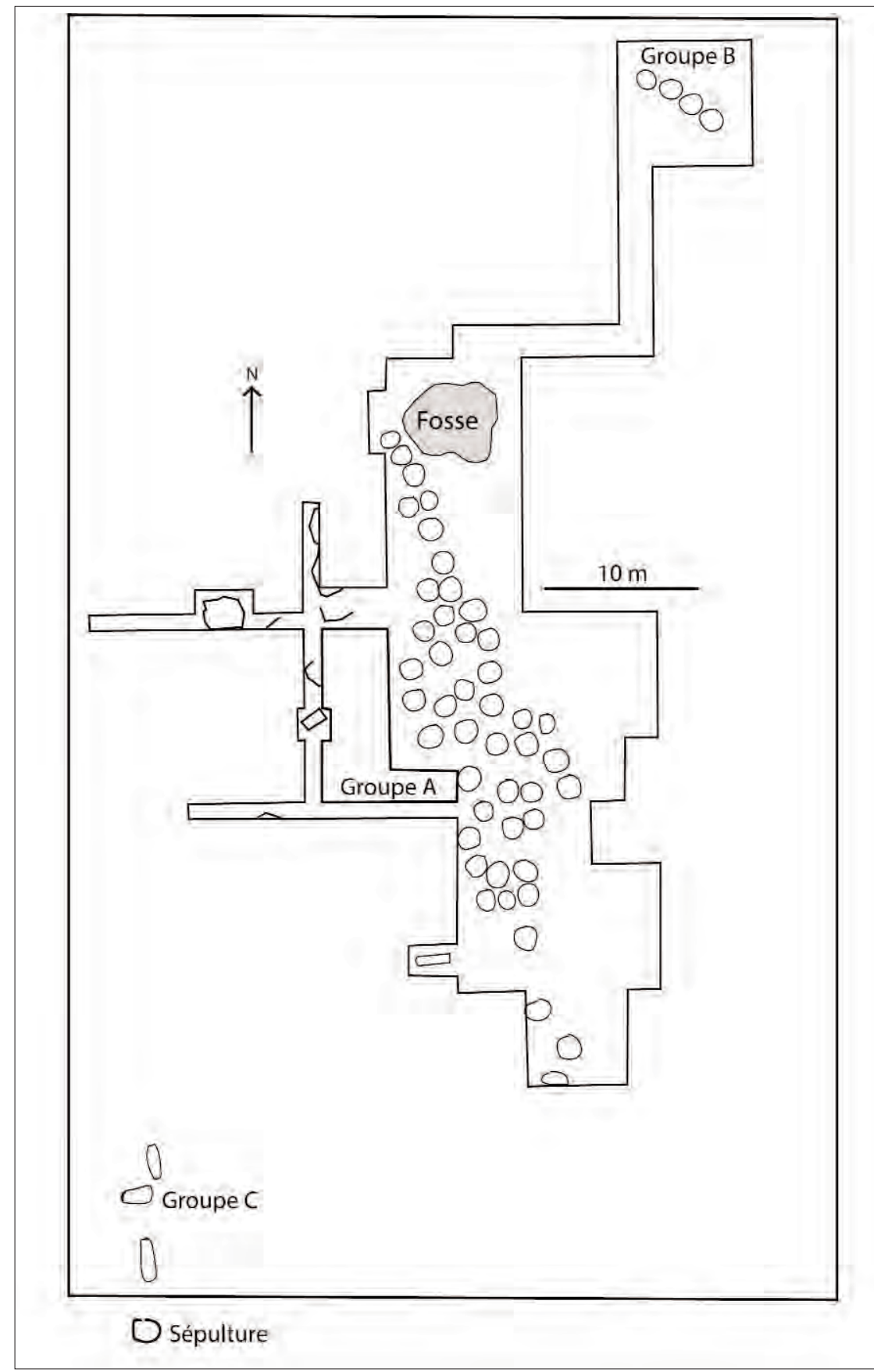

Figure 2 - Plan d'ensemble des fouilles avec emplacement des trois groupes de stèles, des tombes et de la Fosse (d'après Joussaume 1995).

s'étend sur l'ensemble du site et dans laquelle ont été excavées les tombes. Il s'agit d'une terre argileuse, rouge et stérile.

Une date ${ }^{14} \mathrm{C}$ réalisée sur charbon (Gif. 8786) a donné : 820 \pm 60 B.P., soit en date calibrée : 1049 - 1270 après J.-C. Ce résultat est tout à fait compatible stratigraphie de la fosse ont livré une couche superficielle de terre grise, puis les $40 \mathrm{~cm}$ suivants, une terre noire où sont présents des tessons abrasés, des blocs erratiques portant pour la plupart des traces d'éclatement et de rubéfaction dues au feu, des éclats d'obsidienne, des ossements d'animaux et quelques rares charbons de bois.

Ce n'est qu'à $60 \mathrm{~cm}$ de profondeur que se dessine clairement le bord de la fosse, creusée dans la terre rouge déjà citée. Dans la fosse, la terre est argilo-sableuse avec des pigmentations ocre résultant peut-être de la décomposition des conglomérats et des rhyolites. Le mobilier est le même que dans les niveaux supérieurs avec, toutefois, une réduction de l'abrasion des tessons et une augmentation du nombre d'ossements. À $110 \mathrm{~cm}$, au fond de la fosse, on observe «un groupe de blocs en place, posés à plats, restes probables d'une structure de cuisson» (Lagasquie, 1995 : 277)

Le mobilier est donc essentiellement composé de tessons de poterie et de restes fauniques. Outre cela, les vestiges comprennent quelques pièces en obsidienne, dont certaines ont été retouchées en grattoirs, quelques perles en verre et cornaline de même qu'une tige et une lame de fer provenant peut-être d'une épée mais de morphologie différente de celles représentées sur les stèles. La poterie exhumée de la fosse se compose de récipients fermés et ouverts. Ils sont souvent noirs, bien lissés et présentent une grande variété de formes, de moyens de préhension et de décors. Parmi ces derniers on peut observer des cannelures simples ou multiples, cordons lisses ou impressions, estampages divers, petits boutons,... 


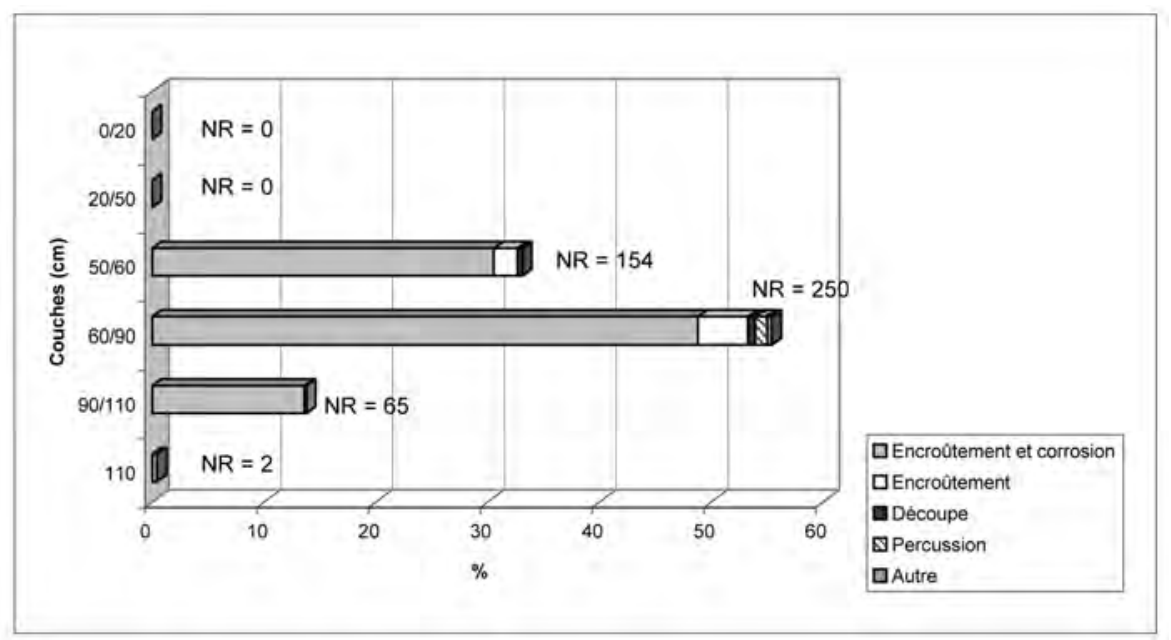

Figure 3 : Répartition des restes fauniques et des traces d'origine anthropique et naturelle dans les différents niveaux de la Fosse.

\section{LOCALISATION ET ÉTAT DE CONSERVATION DES RESTES}

Les vestiges osseux sont nombreux (502 restes) et se répartissent essentiellement dans la partie centrale et profonde de la fosse entre $50 \mathrm{~cm}$ et $110 \mathrm{~cm}$ avec une majorité entre 60 et $90 \mathrm{~cm}$ (figure 3). Comme on peut le voir sur le tableau 1, plus de $90 \%$ des restes présentent des traces d'encroûtement associées dans plus de $80 \%$ des cas à une corrosion très marquée. Ce double phénomène explique en partie les mauvaises performances de détermination car seuls $25 \%$ des vestiges ont pu être attribués à un taxon et à une région squelettique. De plus, la dissolution superficielle du cortex osseux, due à la forte corrosivité du sédiment, a certainement effacé les traces d'origine anthropique telles que celles de découpe ou de percussion. En effet, on remarque que respectivement seuls $1,6 \%$ et $0,9 \%$ des os portent de telles marques. De façon beaucoup plus sporadique, quelques vestiges portent les marques de grignotage, probablement de rongeurs, et de racines.

Enfin, un fait beaucoup plus surprenant est l'absence complète de trace de brûlure. Nous avons vu en effet que la fosse comporte en son centre une structure de combustion et que de nombreux vestiges tels que les blocs éclatés et rubéfiés et les charbons témoignent d'une importante action du feu. Il apparaît donc que les os n'ont pas, quant à eux, été exposés directement au feu. Si, comme le suggère Lagasquie (1995, p.285), la fosse a "été conçue pour la réalisation... d'une structure de cuisson qui aurait essentiellement servi à la consommation de bovinés", ce mode de cuisson a été indirect sans exposition des os à la flamme, qui n'ont été déposés dans la fosse qu'après son refroidissement.

\section{CARACTÉRISATION DES BOVINS}

Sur les 502 restes osseux présents dans la fosse, seuls 103 ont pu être déterminés et tous se rapportent au bœuf (Bos sp.). L'attribution spécifique au bœuf taurin sans bosse (Bos taurus)

\begin{tabular}{|l|r|}
\hline NR & 502 \\
PdR & 3661 \\
NRIPdR & 7,3 \\
\hline$\%$ NR Indet. & 74,3 \\
$\%$ NR Esquilles & 42,8 \\
$\%$ Corrosion & 80,7 \\
$\%$ Encroûtement & 94,2 \\
$\%$ Traces de découpe & 1,6 \\
$\%$ Traces de percussion & 0,9 \\
$\%$ Traces de racine & 0,2 \\
$\%$ Traces de morsure & 0,2 \\
$\%$ Traces de brülure & 0 \\
\hline
\end{tabular}

NR, Nombre de Restes ; PdR, Poids des Restes (en grammes) ; Indet, Indéterminés ; Esquille, tout fragment non attribuable à une pièce anatomique

Tableau 1 - Profil taphonomique de l'assemblage faunique (d'après les critères de Vigne, 1996).

ou au zébu (Bos indicus) n'a cependant pas été possible. En effet, seuls quelques d'ossements permettent leur distinction et aucun n'est présent ici (Grigson, 2000).

D'après les données archéozoologiques actuelles (figure 1), il semble que le premier bovin présent dans la Corne de l'Afrique soit un bœuf taurin sans bosse (Bos taurus). On le trouve de façon assurée vers 
3500 BP sur le site d'Asa Koma à Djibouti (Guérin et Faure, 1996 ; Lesur, 2004). Deux autres sites situés en Ethiopie et datés de la même époque, ont également fourni des restes de bœuf. Il s'agit des sites de Lake Besaka (Brandt, 1980) et de Laga Oda (Clark et Prince, 1978, Clark et Williams, 1978). Cependant, dans les deux cas, les échantillons de faune sont très réduits et la détermination a été réalisée sur des fragments de dents, ce qui limite fortement la fiabilité de ces résultats. En comparaison, dans les régions périphériques de la Corne, les restes de bovins proviennent de sites beaucoup plus anciens. Ainsi, on le trouve dès le 6e millénaire $\mathrm{BP}$ au Soudan, vers 4000 BP au Kenya (Marshall, 2000) et dès le 5e millénaire avant J.-C. au Yémen (Cleuziou et al., 1992).

Le zébu (Bos indicus) arrive quant à lui beaucoup plus tardivement. La plus ancienne preuve de sa présence dans la Corne provient d'une figurine de bronze trouvée sur le site de Zeban Kutur et datée du début de la période aksumite (vers le $2^{\mathrm{e}}$ siècle après J.-C.; Ricci, 195558). Un autre de site de la même période, celui de Matara, a également livré une figurine de bœuf à bosse (Anfray, 1967). Au niveau ostéologique, aucun ossement archéologique n'a pu être attribué au zébu. En Egypte, les représentations dans les tombes et les temples attestent de sa présence dès le $2^{\mathrm{e}}$ millénaire avant J.-C. (Nicolotti et Guérin, 1992).Plus au sud, au Kenya, les premiers restes proviennent de sites récents, datés du $1^{\mathrm{er}}$ millénaire de notre ère (Marshall, 2000).

Les deux espèces sont donc potentiellement présentes à Tiya. Il s'agit peut-être même d'une hybridation entre les deux appelée communément sanga. Ce phénomène, en effet, est à l'origine de la plupart des races bovines africaines actuelles et est probablement survenu de façon assez précoce dans cette région d'Afrique puisqu'elle apparaît, d'après les données génétiques (Loftus et Cunningham, 2000), comme le foyer de diffusion du sanga. Cependant, dans le cas présent, seule l'analyse de l'ADN fossile des os permettrait de trancher la question.

Quoiqu'il en soit, au moins quatre bovins composent l'assemblage de la fosse de Tiya. L'existence de trois chevilles osseuses, dont les bases sont relativement bien préservées semble indiquer qu'au moins un mâle et un bœuf sont présents. En effet, une des chevilles osseuses présente une section forte et circulaire, typique des individus castrés et deux autres (une droite et une gauche, probablement du même individu) une section ovale, typique des mâles (Chaix et Méniel, 1996). Il faut toutefois souligner que la méthode de distinction des sexes par la cheville osseuse est très discutable, surtout dans le cas de race africaine, probablement croisée avec du zébu dont les caractéristiques des cornes n'ont pas encore été clairement définies. Il est possible d'estimer l'âge d'abattage des animaux par l'observation des restes dentaires mais ici seuls 3 individus sont représentés par leurs dents. Les résultats montrent la présence de trois individus adultes (de plus de 36 mois) ${ }^{1}$. Ces informations morphoscopiques concernant l'âge et le sexe des animaux sont néanmoins limitées et sujettes à discussion.

Pour tenter de caractériser un peu mieux la morphologie de ces bovins, nous avons eu recours à l'ostéométrie pour tous les fragments mesurables provenant d'animaux adultes (Annexe 1) ${ }^{2}$. Actuellement, la race de bovin dominante dans cette région d'Éthiopie est la race Arsi, petit zébu (110 cm au garrot en moyenne) à cornes courtes qui est principalement élevé pour la viande (Alberro et Hailé-Mariam, 1982 ; Faye, 1990). Malheureusement, nous n'avons aucune donnée ostéologique bibliographique sur cette race et le matériel de Tiya est trop fragmenté pour permettre une estimation de la stature de ces bovins. Il est donc pas possible pour l'instant de comparer les individus actuels et archéologiques et de suivre l'évolution des bovins dans cette région.

En revanche, les données métriques apportent quelques résultats complémentaires concernant le sexe des animaux. En effet, le diagramme bivarié réalisé à partir des extrémités proximales des radius ${ }^{3}$ (figure 4), montre que parmi les quatre individus représentés (un des individus ayant livré deux os mesurables), l'un semble beaucoup plus gracile que les autres et pourrait donc correspondre à une femelle. Un autre individu semble, quant à lui, beaucoup plus robuste que les autres et pourrait alors représenter le mâle mis en évidence précédemment. Enfin, les deux autres bovins, de format moyen, correspondraient alors à des animaux castrés dont un a été reconnu par la cheville osseuse. Toutefois, ces résultats sont à prendre avec beaucoup de précautions puisque nous ne raisonnons qu'à partir d'un seul type de mesure et de seulement quatre individus, ce qui limite fortement la validité de toute interprétation. 
Finalement, le site de Tiya a livré quatre bovins dont il est encore difficile de définir les caractéristiques morphologiques. Cependant, nous pouvons déjà noter que des individus de différents sexes sont représentés puisque nous trouvons à la fois une vache, deux bœufs et un taureau. Par ailleurs tous étaient adultes au moment de l'abattage.

\section{Origine de L'ASSEMB LAge}

$\mathrm{Au}$ vu du matériel archéologique trouvé en association avec les restes fauniques au sein de la fosse et de la configuration de cette dernière, l'origine anthropique des ossements de bovins ne fait aucun doute. Le mode d'utilisation de ces animaux est cependant plus difficile à définir.

Suivant la méthode de Dodson et Wexlar (1979), nous avons calculé le profil de représentation des parties squelettiques pour tenter d'estimer la conservation (résultant d'un biais taphonomique ou d'une sélection anthropique) des différents éléments anatomiques. Le profil ainsi réalisé (figure 5), nous observons une relativement bonne représentation des éléments crâniens (surtout de la mandibule) ainsi que des membres antérieurs et postérieurs à l'exception du fémur complètement absent. Plusieurs facteurs peuvent expliquer cette carence. D'une part, la dégradation taphonomique due notamment à la corrosion a pu provoquer la fonte et la disparition des éléments osseux les moins résistants dont fait partie l'épiphyse distale du fémur (Brain,
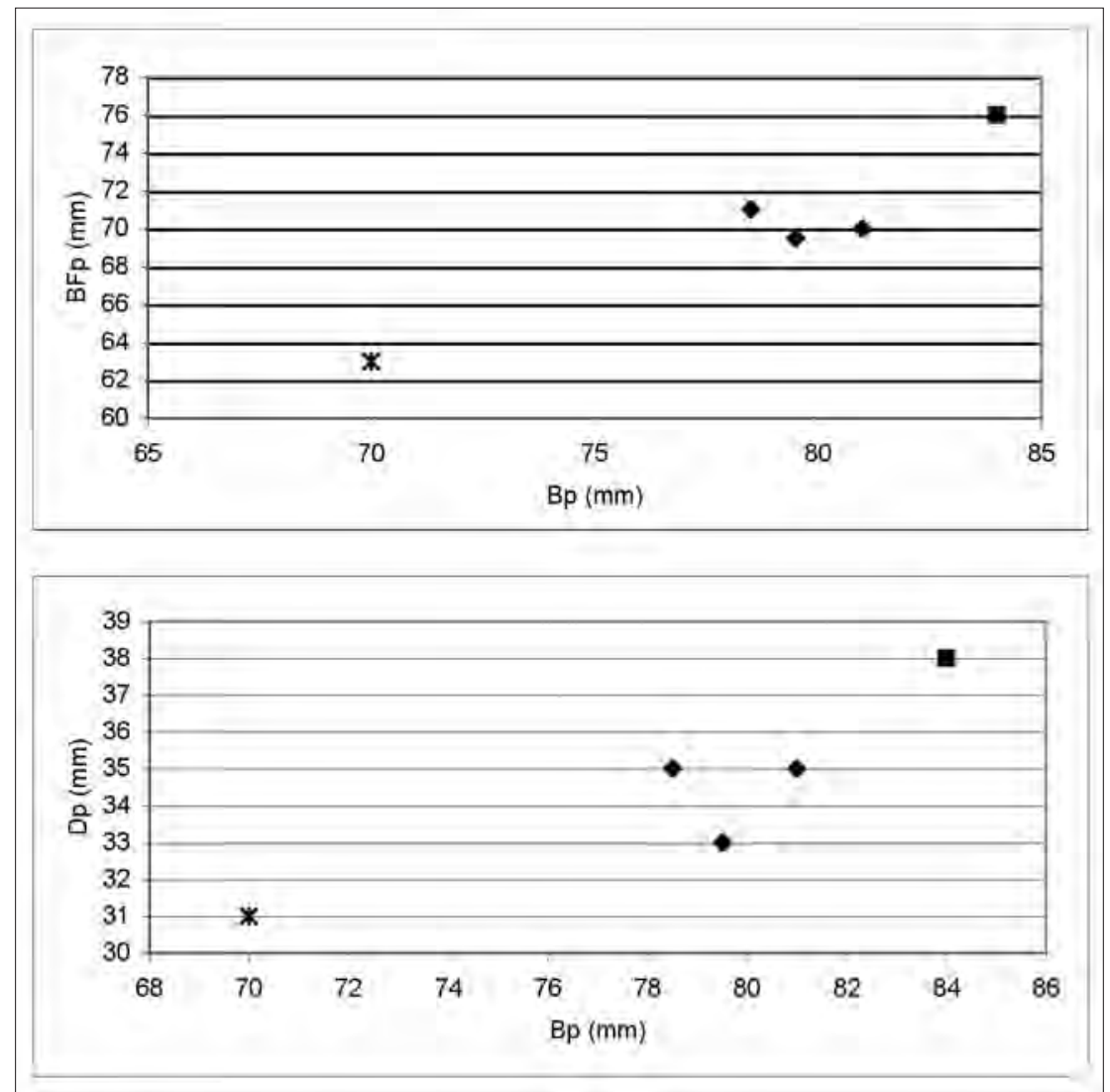

Mesures en millimètres d'après Driesch (1976) : DFp = Breath of the Facies articularis proximalis $; B p=$ Breath of the proximal end et $D p=$ Depth of the proximal end

Figure 4 - Diagrammes bivariés présentant les données métriques des radius proximaux peut-être attribuables à une vache (étoile), deux bœufs (losanges) et un taureau (carré).

1981). Ce processus expliquerait également le fort déficit en vertèbres. D'autre part, l'importante fragmentation a limité l'attribution anatomique d'un grand nombre de fragments de diaphyses parmi lesquelles peuvent se trouver celles du fémur. Toutefois, les processus postdépositionnels ne sont pas uniquement responsables de l'aspect de ce profil qui reflète également l'action de l'homme. On note en effet une absence totale des extrémités (carpe, tarse et phalanges) qui sont d'une

1 - L’estimation de l'âge a été réalisée selon la méthode de Ducos (1958).

2 - Les mesures ont été prises selon les critères de Driesch (1976).

3 - Cet os ayant fourni le plus grand nombre de framents mesurables.

manière générale des éléments résistants (Brain, 1981). Une première explication de ce phénomène pourrait être un problème lors de la fouille avec un ramassage ne portant que le matériel volumineux comme les os longs. Toutefois, la présence d'artefacts de petite taille comme les perles témoigne du "décapage minutieux» de la fosse (Lagasquie, 1995). Si cette absence ne résulte pas d'un biais méthodologique, elle atteste donc de comportements différentiels lors de la constitution de cet assemblage.

Pour les phalanges, cette situation est typique d'un processus de récupération des peaux où ces os restent souvent bloqués 


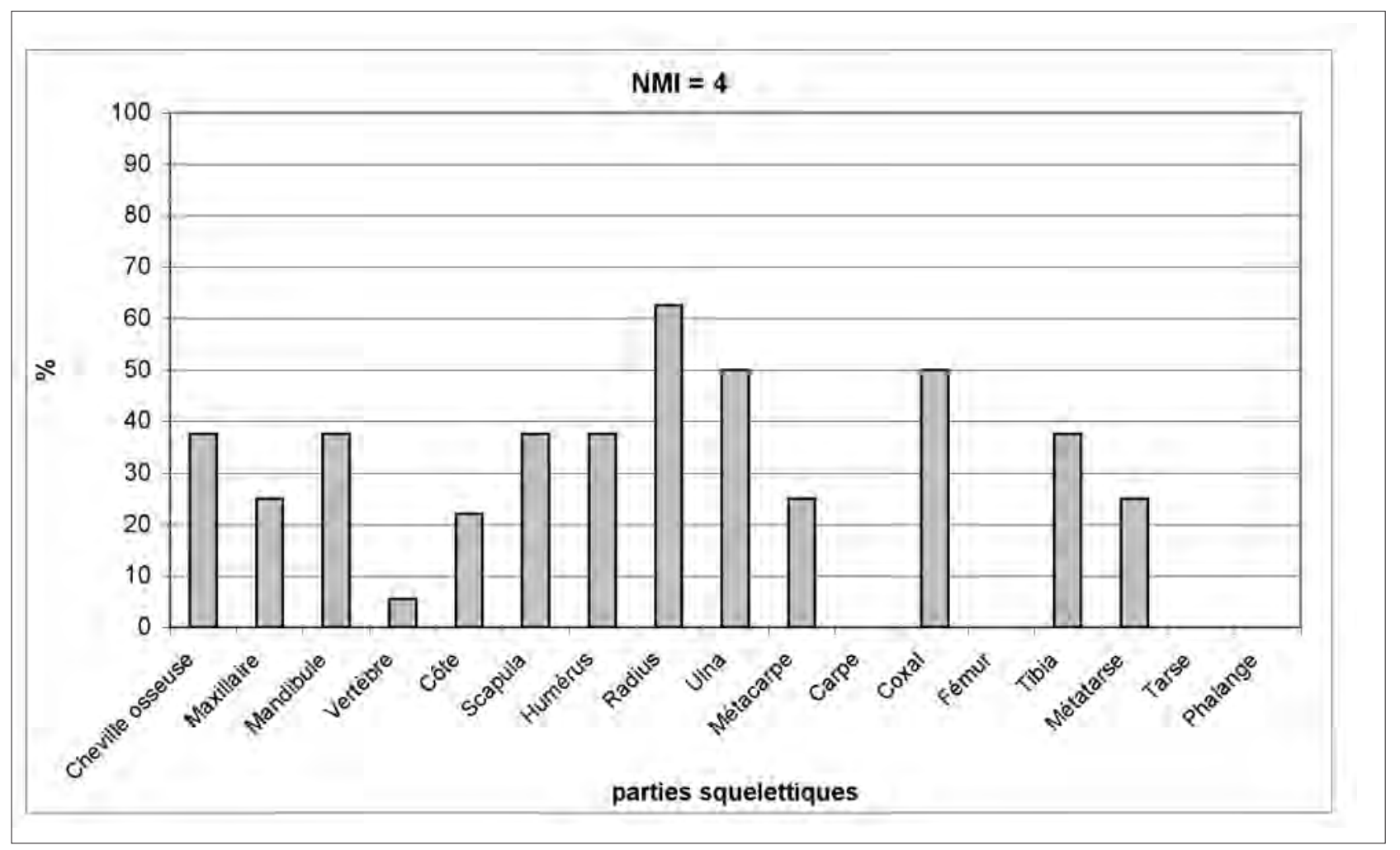

Figure 5 - Profil de représentation des parties squelettiques, calculé selon la méthode de Dodson et Wexlar (1979).

jusqu'au moment du tannage. Pour les carpes et tarses, l'interprétation est plus délicate puisqu'une partie au moins des métapodes est présente. On peut alors supposer que les hommes de Tiya ont eu un intérêt particulier à conserver ces petits os qui ont pu être transportés dans un autre lieu. A titre de comparaison, on peut ainsi citer les Grecs qui utilisaient les talus de moutons au cours de jeux ou de certaines pratiques divinatoires (Poplin, 1992). Il est également possible d'imaginer qu'au contraire, ces os ont été rejetés hors de la fosse pour leur manque d'utilité. On observe en effet dans le profil une domination des parties charnues des membres qui semblent indiquer une utilisation alimentaire des animaux. Dans ce même cadre, les os comme les métapodes mais aussi les mandibules qui ne portent pas de viande mais sont riches en graisse et moelle, ont pu être récupérés pour leur intérêt nutritif, ce qui n'est pas le cas des os du carpe et du tarse. Enfin, nous pouvons envisager que cet assemblage résulte d'un dépôt secondaire, comme les rejets d'un repas réunis en bloc dans la fosse qui a servi à le cuire. Dans ce cas, seuls ont été ramassés les gros éléments, les petits étant restés sur place, hors de la fosse.

Si l'on regarde à présent les traces anthropiques laissées sur les os, de nouveaux éléments apparaissent quant au mode de préparation des animaux. Il faut toutefois signaler qu'en raison de l'action fortement corrosive du sédiment, peu de traces sont encore visibles et seules six ont $\mathrm{pu}$ être observées. Quatre d'entres elles, situées sur le rachis, semblent correspondre à la découpe des carcasses alors que les deux autres observées sur les diaphyses d'ulna et de radius attestent de l'enlèvement des ligaments et de la viande. De plus, les quelques traces dues aux percussions observées sur la diaphyse de certains os $(0,9 \%)$ témoignent d'une fracturation des os longs probablement dans le but d'une récupération de la moelle.

Finalement, la présence de quasiment tous les éléments squelettiques témoigne d'un apport des animaux complets aux abords de la fosse. Nous ne pouvons cependant pas déterminer si les bovins étaient apportés vivants ou s'ils étaient abattus dans un autre lieu. Les traces laissées sur les os attestent d'une préparation des carcasses sur place avec l'enlèvement des cer- 
tains os comme les extrémités probablement pour la récupération de la peau ${ }^{4}$. Le reste des éléments semble avoir été utilisé à des fins alimentaires.

Le mode de consommation est plus difficile à percevoir. Nous avons vu en effet que la fosse contenait des restes de structures de combustion, alors qu'aucun des os ne présentait de trace de brûlure. Il semble donc que les quartiers de viande n'aient pas été rôtis. Au vu de ces données d'apparence contradictoire, plusieurs hypothèses peuvent être formulées.

Premièrement, on peut envisager que la totalité des quartiers ait été consommée sur place et bouillie au sein de récipients prévus à cet effet. Cela expliquerait alors que les os n'aient pas été en contact direct avec le feu.

Deuxièmement, la viande une fois ôtée des os a pu être consommée crue comme c'est encore la tradition dans certaines régions d'Ethiopie. Peu d'éléments sont disponibles quant à l'origine de ce mode alimentaire mais il est possible qu'il fût déjà pratiqué à cette époque.

Troisièmement, nous pouvons envisager que la viande ait été séparée de la carcasse, puis partagée entre les personnes présentes et que seul les os aient été bouillis pour en tirer la moelle et la graisse. Cette pratique est encore attestée dans certaines sociétés éthiopiennes comme les Konsos (Hallpike, 1972). Lors de certaines cérémonies, un taureau est sacrifié, puis la viande est partagée entre les officiants, qui la consomment alors dans l'enceinte familiale, hors du lieu sacrificiel. Seules certaines parties de l'animal sont consommées durant la cérémonie. Dans cette même optique, on peut imaginer que la peau de l'animal était un des éléments à partager.

Enfin, la dernière hypothèse serait que la structure de combustion ait eu une autre fonction que celle de la cuisson des animaux. Par la suite, la fosse a alors pu être réutilisée comme dépotoir pour des restes osseux après une consommation dans un autre lieu. A quelques centaines de mètres au sud du cimetière de Tiya des prospections ont été effectuées sur le site de Kéré (Barbier, 1995). L'abondante céramique récoltée sur ce lieu laisse supposer une occupation intense, fort probablement un habitat. Ce matériel présente de nombreuses affinités avec celui de Tiya. Nous pouvons donc envisager que si ce site correspond bien aux habitations des utilisateurs de Tiya, ces derniers aient pu apporter des carcasses ou des quartiers de viande provenant des bovins de la Fosse. Toutefois, si une partie de la viande a pu être consommée de façon ponctuelle sur ce site, il est difficile d'envisager qu'il y ait eu un réel lien entre la fosse et les habitations, les deux étant relativement éloignées les unes des autres. Par ailleurs, les éventuelles habitations de Kéré devaient déjà posséder des structures d'abattage et de préparation des animaux, ne nécessitant pas l'utilisation de la Fosse de Tiya.

4 - Cette dernière technique est d'ailleurs confirmée par la présence de grattoirs au sein du mobilier.

5 - C'est d'ailleurs encore le cas dans de nombreuses sociétés éthiopiennes comme les Gurage ou les Amharas.

\section{CONCLUSION ET COMPARAI- SONS}

Finalement, tous ces éléments indiquent que l'origine de cet assemblage est principalement alimentaire. Sa proximité géographique et chronologique avec les tombes suggère qu'un lien existe entre ces structures mais il est difficile de définir avec précision lequel. Il est toutefois possible d'envisager qu'à la mort d'une des personnes enterrées dans ce cimetière, un bovin était sacrifié puis consommé sur place ${ }^{5}$.

Le choix de bovins n'est pas sans signification. En effet, depuis les débuts de leur domestication et de leur élevage dans le monde, on trouve des représentations ou des restes de bovidés dans des contextes cultuels et funéraires, comme à Çatalhöyük (Mellaart, 1967) ou Kerma (Chaix, 1990). La symbolique des bovins est souvent liée à la fécondité et à la virilité. Les représentations peuvent être très variées : peintures, figurines modelées, dépôts de bucranes autour de tombes comme à Kerma ou encore sacrifices. À ce propos, il semble que "le sacrifice peut avoir pour objet la récupération d'une peau servant de linceul, ou de procurer de la nourriture au défunt, comme viatique, aux participants de la cérémonie lors d'un banquet funéraire " (Chaix et Méniel, $1996: 91$ ).

L'aspect consommation a déjà été évoqué et semble être confirmé par les données fauniques vues précédemment, mais aussi par la présence de céramique au sein de la fosse qui a pu servir à la cuisson de la viande ou des os. Lutilisation de linceul est, quant à elle, suggérée par plusieurs éléments provenant de la fosse, mais également du cimetière lui même. Tout d'abord nous avons 
vu que le manque complet des phalanges pouvait être un signe de récupération de la peau en vue d'un tannage. De plus, la présence de grattoirs au sein de la fosse est un autre signe de la préparation de ces peaux. Enfin, lors de la fouille des sépultures, plusieurs indices (position contractée forcée de quelques individus, non-effondrement du gril costal dû à cette position et dépôts noirâtres autour de certains corps) ont suggéré que les corps étaient enveloppés dans un sac de matière périssable (Joussaume, 1995). Tous ces éléments laissent donc fortement penser à l'utilisation des peaux pour l'enveloppement des corps. Ils semblent également confirmer l'idée que ces restes de bovins sont à lier à l'utilisation du cimetière et non à d'éventuelles habitations des utilisateurs de Tiya.

Par ailleurs, le choix de l'âge et du sexe des individus est lui aussi peut-être à mettre en relation avec la population présente dans les tombes. Il semble en effet que seuls les adultes aient eu droit à une tombe à stèle (Joussaume, 1995), de même que seuls des bovins adultes ont été abattus. De plus, au niveau de la symbolique des stèles "celles à triade symbolique doivent être considérées comme masculines alors que d'autres, avec masques aux rayures verticales et seins pendants, seraient celles des femmes aux mérites particuliers." (Joussaume, 1995 : 288). Nous pouvons là faire le rapprochement avec la présence au sein de la fosse, de vache, de taureau et de bœuf, ces derniers étant peut-être associés à une autre catégorie de populations comme les jeunes adultes.

Ces données révèlent l'aspect symbolique du choix des individus abattus. En effet, on observe souvent l'importance du sexe des animaux abattus de façon cérémonielle dans nombre de sociétés africaines, pastorales ou non. Ainsi, chez les Konsos, parmi les bovins, seuls les taureaux seront sacrifiés et cela quelle que soit la cérémonie. Le taureau symbolise en effet le paradigme de l'animal sacrifié, la force et la virilité (Hallpike, 1972). L'aspect rituel se situe donc non seulement dans le choix de l'espèce mais aussi dans la sélection particulière des individus qui sont peut-être en association avec les occupants des tombes à stèle. Evidemment d'autres éléments ont pu être pris en compte comme la couleur de la robe ou la forme des cornes dans le choix des animaux. Malheureusement, nous ne disposons d'aucune donnée pour valider cette hypothèse.

L'interprétation de ces résultats dans le contexte éthiopien est difficile car aucune autre donnée n'est disponible sur cette époque. Il n'est donc pas possible d'établir la moindre comparaison.

En conclusion, les restes de bovins de la fosse de Tiya témoignent d'un abattage et d'une consommation, partielle ou complète, à proximité du cimetière. Il est tentant de mettre ces deux ensembles en relation même si aucune preuve directe n'étaye cette hypothèse. Il pourrait alors s'agir d'une cérémonie sacrificielle liée à l'enterrement d'une personne, dont le but pourrait être la récupération de la peau (comme enveloppe pour les corps ?) et l'attribution de nourriture pour le défunt et les convives, le tout autour d'une symbolique des bovins, très fréquente au sein des sociétés pastorales.

\section{Remerciements}

Je tiens à remercier Roger Joussaume, fondateur du GEPCA (Groupe d'Étude de la Protohistoire de la Corne de l'Afrique) et directeur de la fouille du site de Tiya, pour m'avoir autorisé à étudier les vestiges fauniques de ce site ainsi que pour ses conseils lors de la relecture de ce manuscrit. Ma gratitude va également à Jean-Denis Vigne et Louis Chaix pour leur aide pendant l'étude du matériel. Ce travail s'est effectué dans le cadre de ma participation au GEPCA dont je remercie l'actuel responsable, Xavier Gutherz.

\section{B IB LIOG RAPHIE}

Alberro M. et Haïlé-Mariam S. (1982) - Bovins indigènes d'Ethiopie. $1^{\text {re }}$ partie, Revue mondiale de zootechnie, $n^{\circ} 41$, p.2-40; $2^{\mathrm{e}}$ partie, Revue mondiale de zootechnie, $n^{\circ} 42$, p. 27 34.

An fray F. (1967) - Matara. Annales d'Ethiopie,Vol.VII, p. 33-53.

BARbier S. (1995) - Le site de Kéré. Tiya - L'Éthiopie des Mégalithes; du biface à l'art rupestre dans la Corne de
l'Afrique. Joussaume R. (dir.). C hauvigny : Ed. Chauvinoise, mémoire XI, p. 327-348.

Brain C. K. (1981) - The hunters or the hunted? An introduction to African cave taphonomy. Chicago : Chicago University Press.

BRAN DT S.A. (1980) - Investigation of late Stone A ge occurrences at Lake Besaka, Ethiopia. In : Proceedings of the 8th 
PanAfrican Congress on Prehistory and Quaternary Studies 1977, R. Leakey and B. O got (éd.), N airobi: TILLMIAP, p. 239-243.

Chaix L. (1990) - Le monde animal. In : Kerma, royaume de N ubie. Bonnet C. (éd.). G enève : Editions Tribune, p. 108113.

ChaIX L. et MÉN IEL P. (1996) - Éléments d'archéozoologie. Paris : Éditions Errance.

Clark J.D. et Prin CE G.R. (1978) - Use-wear on later Stone Age microlithics from Laga $\mathrm{O}$ da, Harraghi, Ethiopia and possible functional interpretations. Azania 13, p. 101-10.

C LARK J.D. et W ILLIAMS M.A.J. (1978) - Recent archaeolo gical research in southeastern Ethiopia (1974-1975): some preliminary results. Annales d'Éthiopie,Vol. XI, p.19-44.

C leuziou S., In IzAN M.L. et Marcolongo B. (1992) - Le peuplement pré- et protohistorique du système fluviatile fossile Jawf-Hadramawt d'après l'interprétation intégrée d'images satellite, photographies aériennes et prospections. Paléorient, Vol. 18/2, p. 5-29.

DODSON P. et W EXLAR D. (1979) - Taphonomic investigations of owl pellets. Paleobiology, 5, p. 275-284.

D RIESH A. (von den) (1976) - A guide to the measurement of animal bones from archaeological sites. Peabody Museum, 1. $\mathrm{H}$ arvard University.

Ducos P. (1958) - L'origine des animaux domestiques en Palestine. Mémoires de I'Institut de Préhistoire de I'U niversité de Bordeaux 6, Bordeaux.

Faye B. (1990) - Éleveurs d'Éthiopie. Paris : Éditions Karthala.G rigson C . (2000) - Bos africanus (Brehm)? N otes on the archaeozoology of the native cattle of Africa. In: Blench R. M. \& K. C. MacD onald (éd.). The Origins and Development of African Livestock : archaeology, genetics, linguistics and ethnography. UCL Press, London, p. 38-60.

Guérin C. et Faure M. (1996) - Chasse au chacal et domestication du bœuf dans le site néolithique d'Asa Koma (République de Djibouti). Journal des Africanistes t. 66, $n^{\circ} 1$ 2, p. 299-311.
H Allpike C.R. (1972) - The Konso of Ethiopia : a study of the values of Cushitic people. 0 xford, Clarendon Press.

Joussaume R. (sous la dir.) (1995) - Tiya - L'Éthiopie des Mégalithes; du biface à l'art rupestre dans la Corne de I'Afrique. C hauvigny : Ed. C hauvino ise, mémoire XI.

LAgasquie J.P. (1995) - La Fosse. In: Tiya - L'Ethiopie des Mégalithes; du biface à l'art rupestre dans la Corne de I'Afrique. Joussaume R. (dir.). C hauvigny : Ed. C hauvinoise, mémo ire XI, p. 275-285.

LESUR J. (2004) - Exploitation de la diversité faunique et début du pastoralisme dans la Corne de l'Afrique à l'Holocène : Première approche archéozoologique régionale. Mémoire de Doctorat de I'U niversité de Paris I. Mémoire non publié.

Lo ftus R. et CunN In GHAM P. (2000) - Molecular genetic analysis of African zeboid populations. In The Origins and Development of African Livestock: archaeology, genetics, linguistics and ethnography, R.M. Blench and K.C. MacD onald (éd.). Londres et N ew York UCL Press, p. 251-258.

MARSHALL F. (2000) - The origins and spread of domestic animals in East Africa. In The O rigins and Development of African Livestock: archaeology, genetics, linguistics and ethnography, R.M. Blench and K.C. MacDonald (éd.). Londres et N ew York, U CL Press, p. 191-221.

MellaART J. (1967) - Catal Hüyük. A Neolithic town in Anatolia. Londres :Thames and Hudson.

N ICOLOTtı M. et GuéRIN C. (1992) - Le Zébu (Bos indicus) dans l'Égypte ancienne. Archaeozoologia,Vol.V/1, p. 87-108.

Poplin F. (1992) - Les jeux d'osselets antiques. Les Dossiers d'Archéologie, 168, p. 46-47.

RICCI L. (1955-58) - Ritrovamenti archaeologici in Eritrea, II. Rassegna di studi etiopici 15, p. 48-68.

VIGNE J.D. (1996) - La faune des vertébrés. In :J. G asco (dir), Le Laouret et la montagne d'Alaric à la fin de l'Age du Bronze. Centre d'Anthropologie, E.H.E.S.S.-C.N.R.S., Toulouse, p. 197-221.

\begin{tabular}{|l|r|r|}
\hline Os & SLC & BG \\
\hline Scapula G & 50 & 41 \\
\hline Os & BT \\
\hline Humérus D & 65 \\
\hline
\end{tabular}

\begin{tabular}{|l|r|r|r|r|}
\hline Os & Bp & BFp & Dp & SD \\
\hline Radius-ulna D & 70 & 63 & 31 & \\
Radius-ulna D & 78,5 & 71 & 35 & \\
Radius-ulna G & 79,5 & 69,5 & 33 & \\
Radius-ulna G & 81 & 70 & 35 & 40 \\
Radius-ulna G & 84 & 76 & 38 & \\
\hline
\end{tabular}

\begin{tabular}{|l|r|}
\hline Os & SD \\
\hline Métacarpe G & 27,5 \\
\hline \multicolumn{2}{|l|}{} \\
\hline Os & LA \\
\hline Coxal G & 60.5 \\
\hline
\end{tabular}

\begin{tabular}{|c|c|c|c|}
\hline Os & $\mathrm{Bd}$ & BFd & Dd \\
\hline Tibia G & 65,5 & 47 & 50 \\
\hline Os & $\mathrm{Bp}$ & Dp & \\
\hline Métatarse D & 52 & 45 & \\
\hline
\end{tabular}

Mesures en millimètres d'après Driesh (1976) : SLC = Smallest Length of the Collum scapulae $;$ BG = Breath of the Glenoid cavity $;$ BT $=$ Breath of Trochlea $; B p=$ Breath of the proximal end $; B F p=$ Breath of the Facies articularis proximalis $; D p=D e p t h$ of the proximal end $; S D=S m a l l e s t$ breath of the Diaphysis ; LA = Length of the Acetabulum ; Bd=Breath of the distal end ; BFd $=$ Breath of the Facies articularis distalis ; Dd = Depth of the Distal end.

Annexe 1 : Mesures des ossements de Bos de Tiya. 